\title{
Advances in lower hybrid current drive technology on Alcator C-Mod
}

G.M. Wallace 1), S. Shiraiwa 1), J. Hillairet 2), M. Preynas 2)* W. Beck 1), J.A. Casey 3), J. Doody 1), I.C. Faust 1), E. Fitzgerald 1), D.K. Johnson 1), A.D. Kanojia 1), P. Koert 1), C. Lau 1), Y. Lin 1), R. Leccacorvi 1), P. MacGibbon 1)**, O. Meneghini 1)***, R. Murray 1), R.R. Parker 1), D.R. Terry 1), R. Vieira 1), J.R. Wilson 4), S. Wukitch 1), and L. Zhou 1)

1) MIT Plasma Science and Fusion Center, Cambridge, MA 02139, USA 2) CEA, IRFM, 13108 Saint Paul lez Durance, France 3) Rockfield Research, Las Vegas, NV 89135, USA 4) Princeton Plasma Physics Laboratory, Princeton, NJ 08543, USA

* Current affiliation: Max-Planck-Institut für Plasmaphysik, D-85748 Garching, Germany

** Current affiliation: MKS Instruments, Inc., Wilmington, MA, 01887 USA

*** Current affiliation: General Atomics, La Jolla, CA, USA

E-mail: wallaceg@mit.edu

\begin{abstract}
Lower Hybrid Current Drive is an attractive option for non-inductive tokamak operation due to its high current drive efficiency and ability to drive current off axis. The parameters of the Alcator C-Mod LHCD system $\left(f_{0}=4.6 \mathrm{GHz}, B_{\phi} 5.5 \mathrm{~T}\right.$, $\bar{n}_{e} 10^{20} \mathrm{~m}^{-3}$ ) are similar to the proposed LHCD system on ITER. This paper will describe improvements in LHCD technology on C-Mod designed to increase single-pass absorption at high $n_{e}$, extend pulse length (to $>3 \mathrm{~s}$ ), and increase power delivered to the plasma by reducing reflection coefficients. Modeling of LH wave propagation indicates that the loss of LHCD efficiency at higher $\bar{n}_{e}$ can be mitigated by enhancing the single pass power absorption. To this end, an off mid-plane launcher has been designed combining the 4-way poloidal splitting concept of the current LH antenna on C-Mod with a toroidal bi-junction. The four rows of the launcher are located above the mid-plane in order to exploit the poloidal upshift of $n_{\|}$as rays propagate from the antenna into the plasma. The maximum LHCD pulse length was previously restricted to $0.5 \mathrm{~s}$ to prevent boiling of the klystron collector coolant. The Transmitter Protection System (TPS) was redesigned to model the coolant temperature in real time and shut off the klystron beam if the coolant is close to boiling. The TPS upgrade has been installed and operated on C-Mod for pulses up to $4.5 \mathrm{~s}$ into dummy loads and $1.0 \mathrm{~s}$ into the plasma. A new moveable local LH launcher protection limiter was designed to reduce reflection coefficients across a wide range of launcher positions while still providing effective protection from plasma heat flux. Initial high power $\left(P_{n e t} \sim 700 \mathrm{~kW}\right)$ results show that lower reflection coefficients are achievable with the new limiter configuration as compared to the old configuration. Rapid degradation of power handling after boronization of the plasma facing surfaces, which does not occur with the fixed position limiters, highlights the need for the local protection limiter to provide sufficient protection from contamination during boronization.
\end{abstract}


PACS numbers: 52.35.Hr, 52.50.Sw, 52.55.Wq 


\section{Introduction}

In addition to its highest level scientific objective to obtain an energy gain, Q, of 10, the mission of ITER [1] is also to demonstrate "steady state through current drive at $Q>5 "[2]$. This objective is motivated by the need for a fusion reactor to operate in essentially steady-state conditions, where the lifetime of the components would not be unduly limited by considerations of thermo-mechanical fatigue. Lower Hybrid Current Drive (LHCD) is an attractive option for non-inductive tokamak operation on ITER due to its high current drive efficiency and ability to drive current off axis [3]. The operational parameters of the Alcator C-Mod LHCD system [4] $\left(f_{0}=4.6 \mathrm{GHz}\right.$, $B_{\phi} 5.5 \mathrm{~T}, \bar{n}_{e} 10^{20} \mathrm{~m}^{-3}$ ) are similar to the proposed LHCD system on ITER [3]. This paper documents advances in LHCD technology on C-Mod designed to extend pulse length, reduce reflection coefficients, and increase single-pass absorption at high density. Section 2 describes the design of an advanced, off mid-plane LHCD antenna. Modeling of LH wave propagation indicates that the observed loss of LHCD efficiency at high density can be mitigated by enhancing single pass power absorption. This is achieved by exploiting the poloidal upshift of $n_{\|}$from rays launched above the mid-plane to bridge the spectral gap. Phase space synergy with the rays launched above the midplane results in stronger absorption of the rays launched from the mid-plane. Section 3 covers the implementation of an advanced Transmitter Protection System (TPS). The $0.5 \mathrm{~s}$ pulses achieved in previous operations are sufficiently long as compared to the current redistribution time $\left(\tau_{C R} \sim 0.2 \mathrm{~s}\right)$ for quasi-steady state non-inductive operation, but longer LH pulses are necessary to allow for other plasma parameters $\left(n_{e}, T_{e}\right)$ to reach a new equilibrium once the current profile has been modified. The TPS provides protection to the klystrons by modeling the collector coolant temperature in real-time to prevent boiling of the coolant. The maximum LH pulse length has been extended up to $1.0 \mathrm{~s}\left(\sim 5 \tau_{C R}\right)$ in routine operation with the new TPS. Section 4 compares the performance of an integrated LH protection limiter with a protection limiter fixed to the wall. The integrated limiter moves radially with the LH launcher and is designed to protect the launcher from excessive plasma heat flux across a wide range of radial locations. This allows the launcher to move to a position with the proper density profile to ensure efficient coupling of LH waves from the antenna to the edge plasma. In contrast, the fixed position limiter restricts acceptable launcher positions to a very narrow range, which often results in high reflection coefficients. The integrated limiter reduced reflection coefficients as designed, but at the cost of reduced power handling. The design and testing of a prototype high power double-stub fast ferrite tuner (FFT) for the LHCD system is covered in Section 5. The goal of the FFT is to provide feedback-controlled impedance matching to decrease reflection coefficients and increase net power over a wide range of plasma conditions. Section 6 provides a discussion of the key results. 


\section{Advanced off mid-plane LH antenna}

An above mid-plane launcher has been designed combining the poloidal splitting concept of the current LH launcher on C-Mod [5] with a toroidal bi-junction [6]. The feed waveguide from each klystron is first split 4 ways in the poloidal direction, then two ways in the toroidal direction. Eight splitters are stacked together to form a 16x4 waveguide array as shown in Figure 1. The use of a $90^{\circ}$ bi-junction rather than the now common multi-junction design allows for more control of the launched $n_{\|}$spectrum. Each window is $5.5 \times 60 \mathrm{~mm}$, which gives a launched $n_{\|}$value of 2.33 at $90^{\circ}$ phase difference between adjacent columns. The new antenna was optimized to decrease reflected power and increase directivity over a broad range of plasma conditions and launched $n_{\|}$values. Control over these parameters was accomplished by adjusting the length of the bijunction, the distance between the 4-way splitter and the beginning of the bi-junction, and the differential phasing between poloidal rows. The optimization parameters are shown in Figure 2. The LH coupling code ALOHA [7] was used to determine the scattering matrix of the plasma, which was then coupled to the scattering matrix of the 8-way splitter as calculated with the COMSOL commercial RF simulation code. A linear density profile with a $1 \mathrm{~mm}$ vacuum gap was used in the ALOHA code. The edge density, $n_{0}$, was varied from 2-8 times the cut-off density $\left(n_{c o}=2.7 \times 10^{17} \mathrm{~m}^{-3}\right.$ at $4.6 \mathrm{GHz})$ with density scale lengths $\left(L_{n}\right)$ of $0.1-0.5 \mathrm{~mm}$. The input waveguide differential phasing was varied to scan launched $n_{\|}$over the range of 2.1 to 2.7. Simulated reflection coefficients for the launcher remain nearly constant at $\sim 5 \%$ for the range of plasma conditions considered. Additionally, the directivity of the $n_{\|}$spectrum is insensitive to the launched $n_{\|}$value.

The addition of a second LH launcher on C-Mod will increase the available net LH power from $\sim 1 \mathrm{MW}$ to $\sim 2 \mathrm{MW}$. The higher power will enable non-inductive operation across a wider range of plasma density and current. The two launchers will also allow for exploration of quasi-linear damping synergy between different launched $n_{\|}$values and poloidal launch points. The four rows of the launcher are located above the midplane in order to exploit the upshift of $n_{\|}$as rays propagate from the antenna into the plasma. Ray tracing simulations show that $n_{\|}$upshifts for rays launched above the mid-plane, and downshifts for rays launched from below the mid-plane. The $n_{\|}$upshift results in better wave penetration to the plasma core at higher density $\left(n_{e}>10^{20} \mathrm{~m}^{-3}\right)$ and stronger single-pass absorption of the LH waves launched from above the midplane. Figure 3 illustrates the effect of the phase-space synergy between the mid-plane and above mid-plane launchers. If the rays from the two launchers are considered individually and the LH driven currents are added, the total driven current is $200 \mathrm{kA}$. When the quasi-linear calculation is performed on the full ensemble of rays from both launchers, the LH driven current increases to $300 \mathrm{kA}$. These calculations are discussed in additional detail in [8].

The detailed RF design of the LH3 launcher is complete. Figure 4 shows the result of a finite element simulation of the transverse RF electric fields inside the splitting 


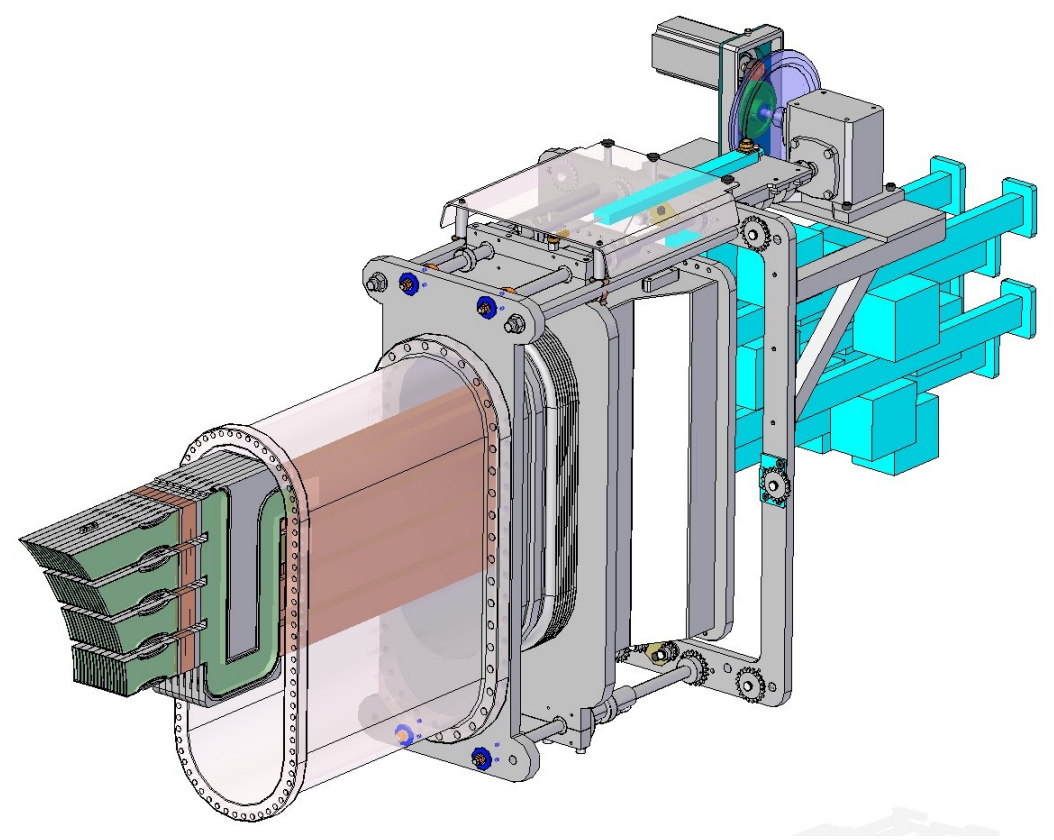

Figure 1. An isometric view of the above mid-plane LH3 launcher design. The vacuum windows are located in copper sleeves between the vertical leg of the 4 -way splitter and the horizontal waveguide containing the final $90^{\circ}$ bi-junction.

Figure 2. A schematic view of the parameters used to optimize the design of the LH3 8-way splitter.

structure and in the edge plasma. The simulation assumes a linear density gradient of ??? $\mathrm{m}^{-4}$ and density at the waveguide aperture of ??? $\mathrm{m}^{-3}$. The four rows of the splitting structure are of different lengths to conform to the shape of the last closed flux surface (LCFS) on C-Mod. The top of each waveguide row is mitered to account for the non-normal angle of incidence between the waveguide and the LCFS. Simulations show that higher order modes are excited at the waveguide aperture if the miter bend is not included in the design. Phase shifters were introduced in the top three rows to optimize the electrical length between the 4-way splitter and the bi-junction septum. Similar phase shifters (shown schematically in Figure 2) are located in one of the two waveguides formed by the bi-junction to set the column-to-column phase shift at 90 . Simulations show that the power splitting between the output waveguides is quite even (-8.6 to $9.4 \mathrm{~dB}$ or 13.8 to $11.5 \%$ per waveguide) and the input S11 is $-20 \mathrm{~dB}$ or $1 \%$ reflected power with matched loads. Alumina vacuum windows are situated between the 4-way splitter and the bi-junctions. These windows can be seen in Figure 4 as the regions of very low electric field in each finger. Each window is $12.5 \times 60 \mathrm{~mm}$ in cross section with an axial length of $\lambda / 2$ for RF transparency. The windows are individually brazed in copper sleeves, as in the LH2 launcher, to reduce the likelihood of stress cracks developing in the windows. Construction of the 8-way splitter will use a similar process 

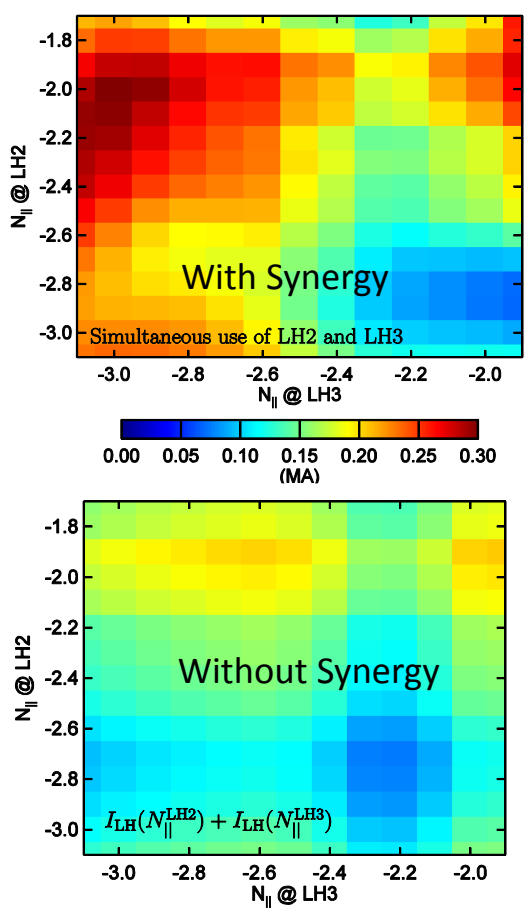

Figure 3. (top) Total LH driven current generated by launchers on the mid-plane and above the mid-plane. (bottom) Total LH driven current for the mid-plane and above mid-plane launchers without considering phase-space synergy.

as in manufacture of the LH2 launcher [5].

Prototype components for the LH3 8-way splitter assembly have been constructed and tested. The final 2-way bi-junction assembly is constructed by milling waveguide channels into either side of a 316? 304? stainless steel plate, resulting in an I-beam shaped cross section. The channels are then copper plated to a thickness of ??? inches to minimize resistive losses in the waveguide. After plating, cover plates are attached to the plate by electron beam welding. Testing of the bi-junction with a network analyzer at low power $(0 \mathrm{dBm})$ shows nearly ideal splitting performance with the two output ports receiving -3.16 and $-3.17 \mathrm{~dB}$ (Figure 5). Phasing between the outputs was measured at $91^{\circ}$ compared to the designed value of $90^{\circ}$. Testing of the bi-junction at high power (up to $233 \mathrm{~kW}$ forward power into matched loads) showed excellent performance with minimal conditioning required. Each bi-junction will be subject to $\sim 50 \mathrm{~kW}$ of forward power (1/4 of the output from a single klystron). It is important to note that these high power tests were conducted with pressurized nitrogen in the bi-junction ( $\sim 8.25$ psi above atmospheric pressure) whereas the bi-junction in LH3 will be entirely under vacuum. Still, it is expected that the bi-junction should withstand 


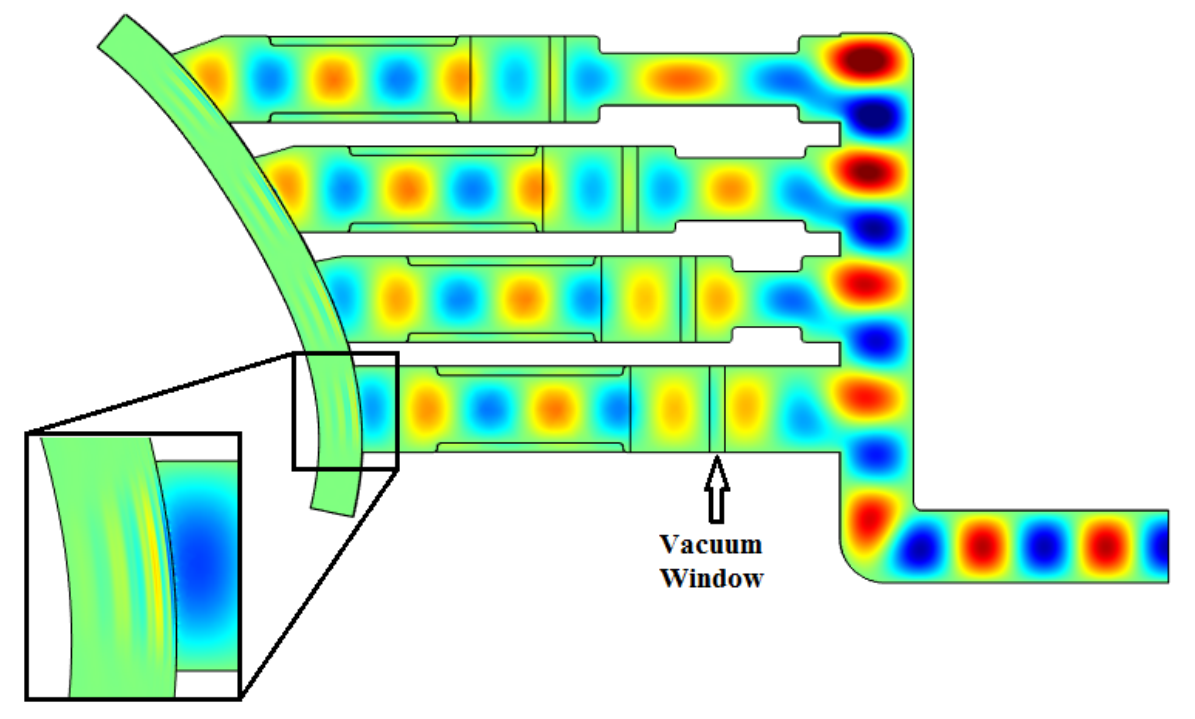

Figure 4. Simulated transverse RF electric fields inside the 8-way splitter and plasma. Short wavelength lower hybrid waves propagate through the plasma from each row of the antenna as shown in the inset.

the nominal maximum forward power of $\sim 50 \mathrm{~kW}$ in vacuum even with $50 \%$ reflected power in the unmatched section of the bi-junction (peak standing wave voltage $=0.8 \times$ nominal voltage at $233 \mathrm{~kW}$ with no standing wave).

The current LH2 launcher is powered by ten klystrons. An additional six klystrons will be added to provide a total of sixteen klystrons split evenly between LH2 and LH3. In addition to the increase in source power, an improved control system will provide better control of the forward power and phase from each klystron. Details of the new control system are presented in [9].

\section{Transmitter protection system}

The maximum LHCD pulse length on C-Mod is limited by heating in the collector of the high power klystron amplifiers. Modeling by the klystron manufacturer shows that the klystron can operate for at least 5 seconds without boiling the coolant with full $\mathrm{RF}$ output power, but the coolant will boil after only $1.2 \mathrm{~s}$ of beam-on time with no RF output power due to the additional beam power dissipated in the collector [10]. Conditions of beam on with no RF output can occur when the Coupler Protection System (CPS) temporarily shuts down the RF drive to the klystron after a fault is detected. These faults sometimes recur continually for the duration of an LH pulse. The maximum pulse length was therefore restricted to durations known to be safe (with 

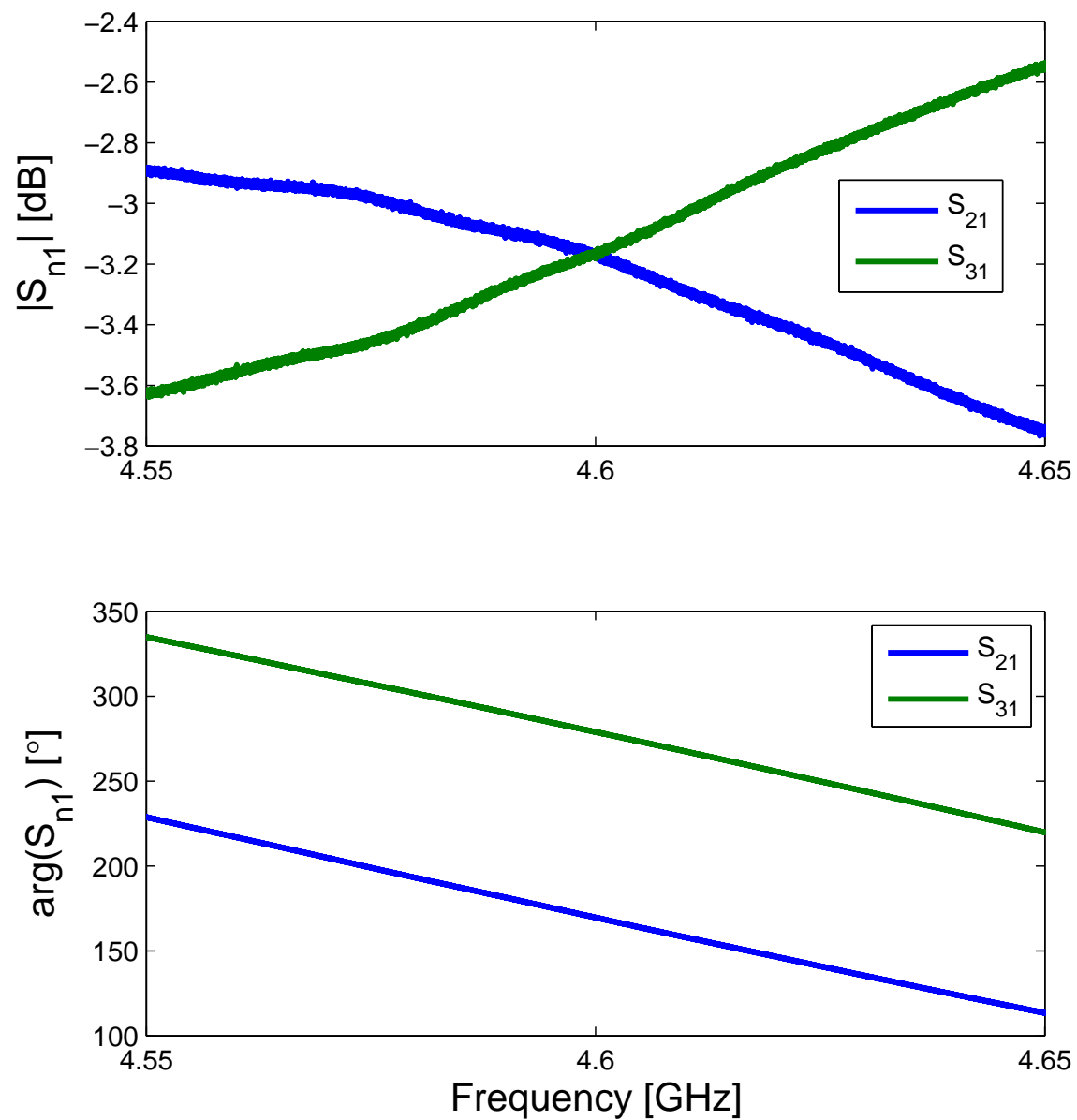

Figure 5. Low power $(0 \mathrm{dBm})$ measurements of the bi-junction prototype component.

some margin) for zero RF output power (i.e. $t_{\text {pulse }}<0.5 \mathrm{~s}$ ). Increasing the LH pulse length to $>1 \mathrm{~s}$ allows for the LH system to remain on for many current relaxation timescales $\left(\tau_{C R} \sim 0.2 \mathrm{~s}\right)$ and extend the flattop length.

The TPS was redesigned to include a collector over-temperature system (COTS) [11]. The purpose of the COTS is to provide a higher level of protection for the collector in the event that the klystron is not operated near full RF output power for a significant period of time $(>\sim 0.5 \mathrm{~s})$. The COTS models the outlet coolant temperature $\left(T_{\text {out }}\right)$ in real time based on the power of the electron beam $\left(V_{b} I_{b}\right)$, the RF output power $\left(P_{R F}\right)$, inlet coolant temperature $\left(T_{i n}\right)$, coolant flow rate $(Q)$, and the heat capacity of the coolant $\left(C_{\left(\mathrm{H}_{2} \mathrm{O}\right)}\right)$, as shown schematically in Figure 6. The COTS microcontroller integrates the heat transfer equation

$$
\frac{d T_{\text {out }}}{d t}=\frac{I_{b} V_{b}-P_{R F}-Q C_{\left(H_{2} O\right)}\left(T_{\text {out }}-T_{\text {in }}\right)}{k}
$$

at a rate of $1 \mathrm{kHz}$. The beam power, $\mathrm{RF}$ output power, and coolant heat flow rate 


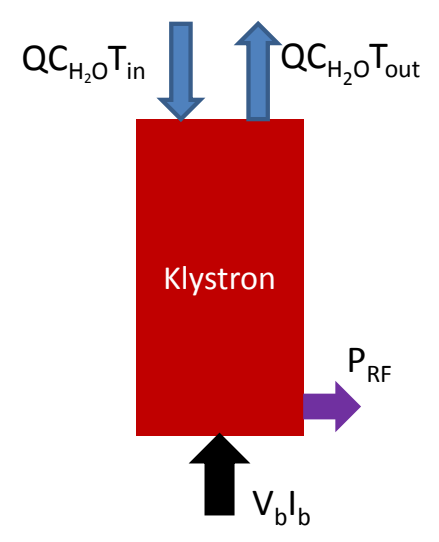

Figure 6. A schematic of the klystron collector system showing power inlets and outlets.

are measured directly. The remaining variable, $k$, is the aggregate heat capacity of the collector in units of $\mathrm{J} / \mathrm{K}$.

A fault condition is generated and the high voltage pulse is terminated if the maximum coolant temperature exceeds a settable safe margin below the boiling point of the coolant at the measured pressure. The maximum coolant temperature is assumed to be proportional to the temperature difference between the inlet and outlet. The constant of proportionality was found to be $\sim 3.6$ based on simulations provided by the manufacturer [10]. If this maximum temperature, $T_{\max }$, exceeds a pre-set margin below the boiling point of water at the operating pressure of $\sim 18 \mathrm{psi}$, then a fault condition is triggered and the high voltage power supply is shut off to protect the klystron. The system is set up such that each of the 16 klystrons is individually monitored and a COTS fault on any one tube will shut down the high voltage power supply (HVPS). All klystrons are connected in parallel to the same HVPS such that a fault on a single klystron will shut down the remaining 15 [12]. Figure 7 shows the rise in coolant outlet temperature during a $4 \mathrm{~s} \mathrm{LH}$ pulse. The solid red curve is the temperature measured at a point downstream of the klystron. This curve exhibits some time lag as compared to the calculated outlet water temperature (blue dot-dashed curve), but otherwise agrees 
very closely in magnitude and time response. The maximum coolant temperature does not reach the fault threshold during this pulse because the klystron was operating with a significant RF output power.

The TPS upgrade has been installed and operated on the C-Mod klystrons. Downstream temperature measurements of the klystron coolant have been made with high temporal resolution (response time $<0.05 \mathrm{~s}$ ) to benchmark the COTS calculation and determine the empirical value for the heat capacity, $k$. The value of $k$ is determined by measuring the decay rate of the downstream outlet coolant temperature (solid red line in Figure 7) for each klystron and adjusting $k$ so that the time constant of Equation 1 matches the downstream measurement. These measurements show a time lag due to the distance between the collector and the downstream measurement location. Despite the time delay, the magnitude of the temperature rise recorded downstream agrees closely with the expected value from Equation 1 . The empirical value $(\sim 35 \mathrm{~kJ} / \mathrm{K})$ is close to the value expected based on the volume of water in the cooling channels combined with the mass of copper in the collector. Pulse lengths of up to $4.0 \mathrm{~s}$ have been demonstrated into dummy loads with no adverse affects.

Figure 8 shows several key plasma parameters during a long-pulse LHCD discharge. In this case $600 \mathrm{~kW}$ of LHCD power is used to drive most of the plasma current for $1.0 \mathrm{~s}$. The loop voltage is reduced from $\sim 1 \mathrm{~V}$ during the inductive phase $(t<0.75 \mathrm{~s})$ to $\sim 0.2 \mathrm{~V}$ during the LHCD phase $(0.75<t<1.75 \mathrm{~s})$. The loop voltage briefly returns to $\sim 1 \mathrm{~V}$ between the end of the LH pulse and the start of the $I_{p}$ rampdown. This LH pulse is $\sim 5 \times \tau_{C R}$ as indicated by the bar in the third panel. The hard x-ray count rate (fourth panel) saturates after $100 \mathrm{~ms}$ and remains elevated until the end of the LH pulse. These longer LH pulses allow for other plasma parameters such as the electron density (second panel) to stabilize before the end of the LH pulse. Longer pulses also allow for more accurate current profile measurements with C-Mods motional Stark effect diagnostic [13].

\section{Integrated LH protection limiter}

A new local LH launcher protection limiter was installed prior to the 2012 run campaign. The previous local protection limiter was fixed with respect to the outer wall of the vacuum vessel. The major radius of the LH launcher could be moved by $\sim 30 \mathrm{~mm}$ with respect to this fixed limiter position, but reflection coefficients were high enough to prevent reliable operation of the LHCD system $\left(\Gamma^{2}>0.5\right)$ unless the launcher radius was very close to the limiter radius $\left(R_{\text {lim }}-R_{\text {launch }}<0.5 \mathrm{~mm}\right)$. A new limiter was designed to reduce reflection coefficients across a wider range of launcher positions while still providing protection from the plasma heat flux. In this new configuration (Figure 9), the LH protection limiter is directly attached to the LH launcher itself and move in and out in unison with the LH launcher.

The tiles of the new limiter protrude beyond the LH launcher by a fixed distance of $0.25 \mathrm{~mm}$. The protection tiles allow the LH launcher to be moved closer to the 


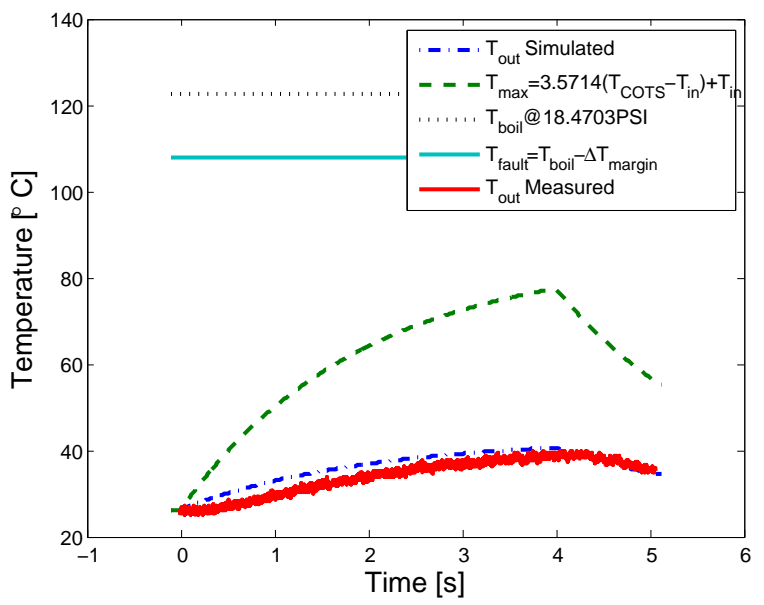

Figure 7. Modeled (blue dot-dashed curve) and measured (solid red curve) outlet temperature rise during a $4 \mathrm{~s} \mathrm{LH}$ pulse. Inlet and outlet temperatures are equal at the start of the pulse. The modeled peak coolant temperature at the copper/coolant interface (green dashed curve) remains below the fault temperature (solid cyan line) for the duration of the $4 \mathrm{~s}$ high power pulse. 


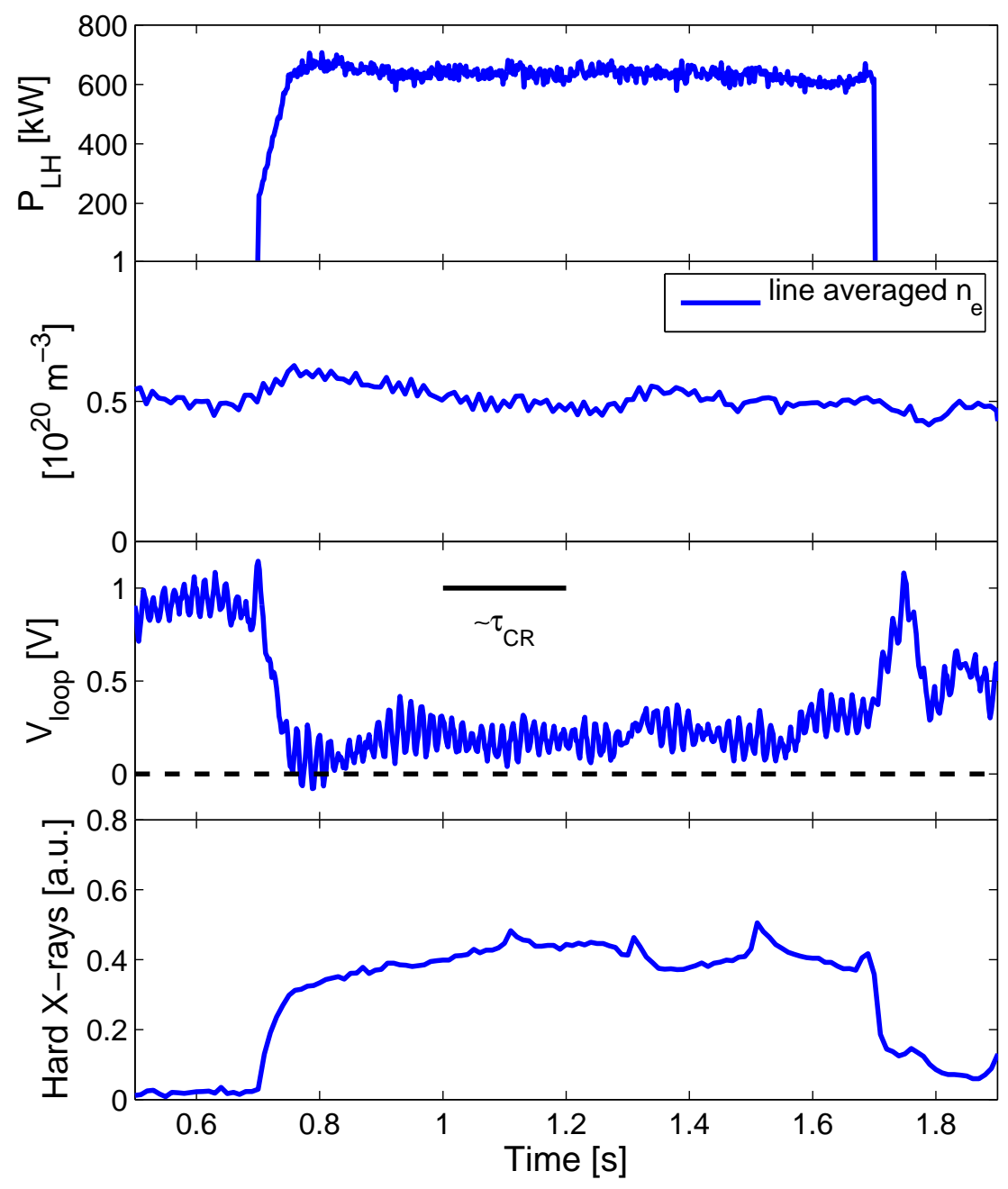

Figure 8. A C-Mod discharge with a majority of the plasma current sustained by LHCD for $1.0 \mathrm{~s}$.

plasma than previously possible without exposing the launcher to the full heat flux of the plasma. Results from Tore Supra show that reflection coefficients can be reduced by moving the LH to a position in front of adjacent ICRF antenna limiters, therefore increasing the field line connection length of the region in front of the LH antenna [14]. Lower reflection coefficients not only increase the available net LH power, but also reduce false positive faults from the VSWR based arc protection system. Trip levels for the arc protection system can thus be set more conservatively, increasing the safety of the launcher. High power $\left(P_{\text {net }} \sim 700 \mathrm{~kW}\right)$ results show that lower reflection coefficients are achievable with the new limiter configuration as compared to the old configuration. Figure 10 shows the distribution of reflection coefficients for the moveable integrated limiter and the fixed position limiter. The most probable, although there is no reduction in the distribution for $\Gamma^{2}$ greater than $\sim 35 \%$. The peak of the distribution shifted 


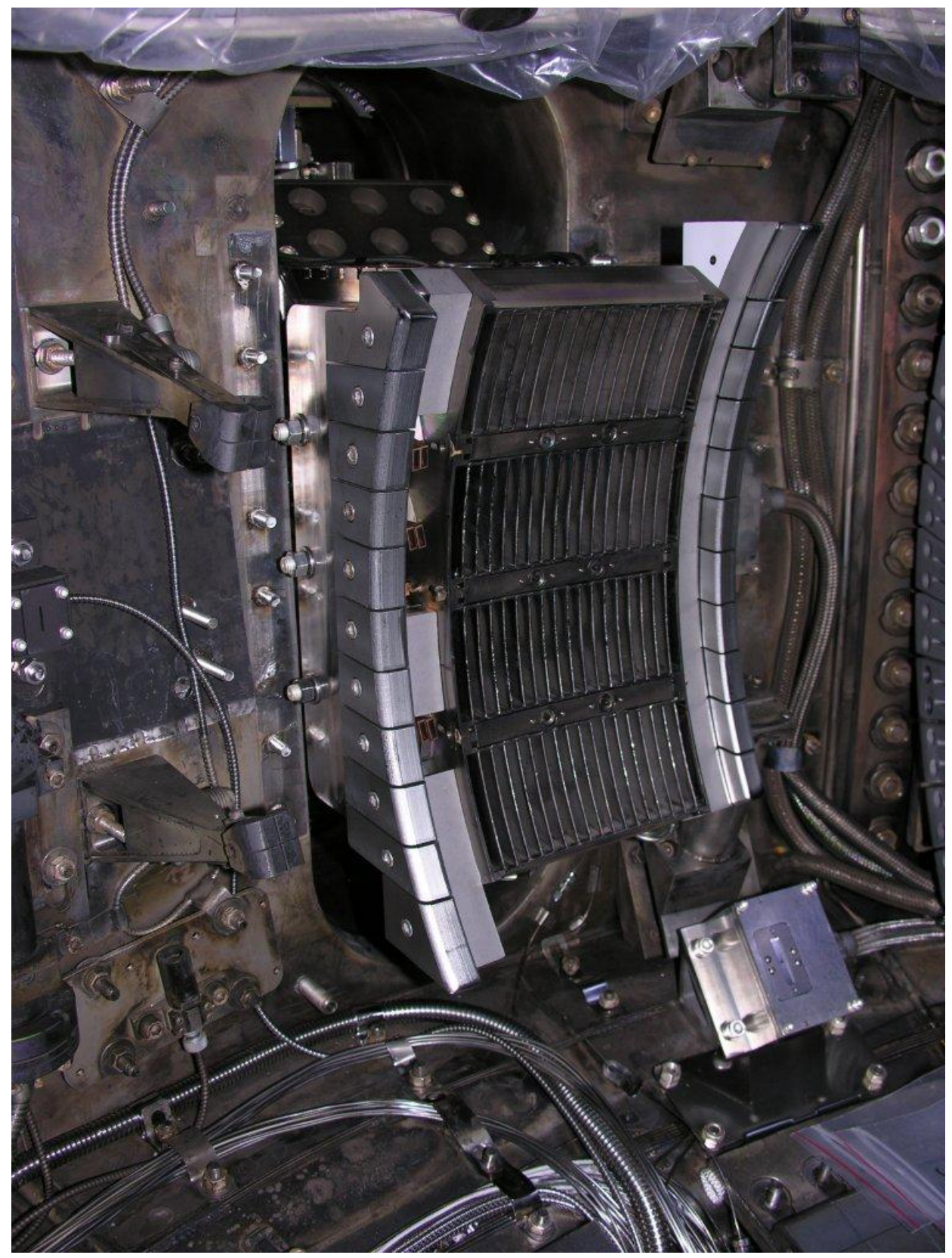

Figure 9. The integrated LH protection limiters as installed on Alcator C-Mod.

from $\sim 29 \%$ with the fixed limiter (dot-dashed green line) to $\sim 24 \%$ with the moveable integrated limiter (solid blue line). For a fixed forward power of $1 \mathrm{MW}$, this represents an increase in net power from $710 \mathrm{~kW}$ to $760 \mathrm{~kW}$, or a $7 \%$ increase in net power.

Although the reflection coefficients were reduced with the integrated limiter, power handling of the launcher fell quickly after an initial period of good performance. Figure 11 shows the net LH power for each discharge with LH during the period with the integrated limiters installed. This drop in power handling may be due to a buildup of boron compounds on the plasma facing surfaces. The first boronization of the 


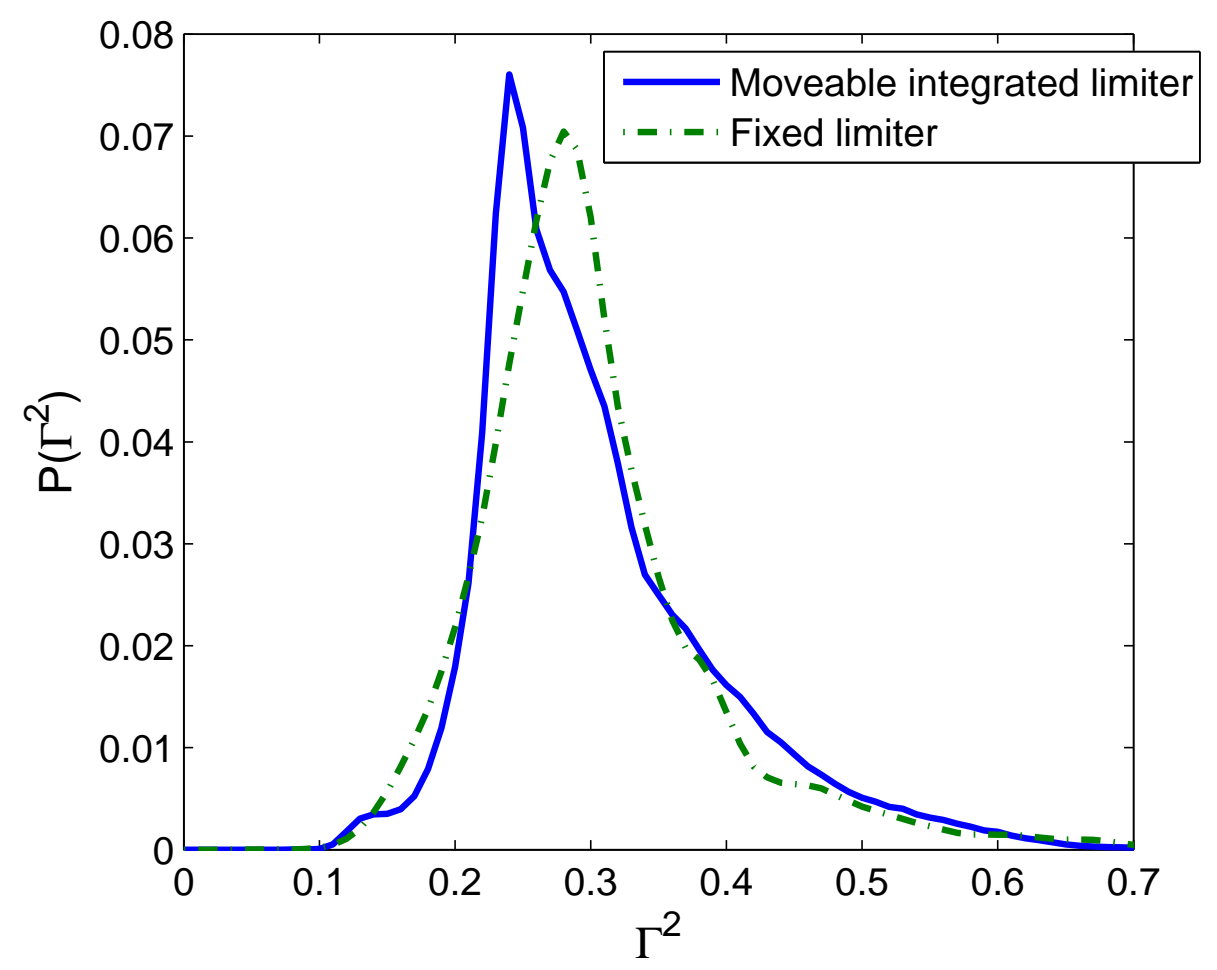

Figure 10. Distribution of reflection coefficients for the moveable integrated limiter (solid blue) and fixed position limiter (dash-dotted green). Distributions are normalized so that $\int P\left(\Gamma^{2}\right) d \Gamma^{2}=1$.

run campaign (a lighter than typical coating) does not appear to have had much adverse effect on performance, but subsequent boronizations substantially reduced the ability of the launcher to withstand high power without arcing. With the fixed limiter, the LH launcher could be retracted $30 \mathrm{~mm}$ behind the limiters. The design of the integrated limiters only allows the launcher to retract $15 \mathrm{~mm}$ from the fully inserted position. In addition, the fixed position limiters provide a deep protected area with very short connection length, effectively shielding the launcher from boron deposition. The integrated limiters only provide a very shallow region $(0.25 \mathrm{~mm})$ of short connection length, and as previously mentioned the launcher cannot be retracted as far as with the fixed position limiters.

Inspection during a manned access showed significant boron deposits on the launcher and arc tracks in several waveguides. The launcher was refurbished (boron film removed and arc tracks polished off) during the manned access and the fixed position limiter was reinstalled. After reinstalling the fixed position limiters, the power handling of the LH launcher recovered to typical levels ( $\sim 800 \mathrm{~kW}$ net power) and has remained there through several hundred high power pulses and frequent boronizations. 


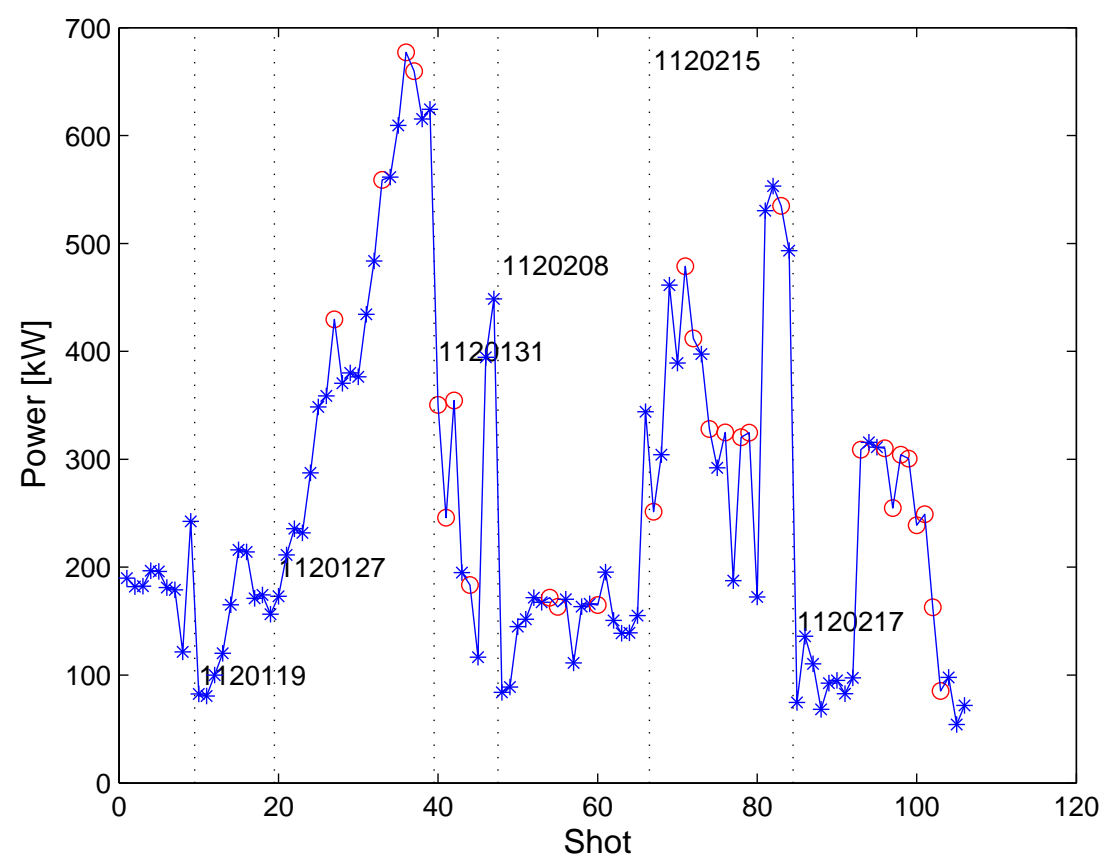

Figure 11. Net power during each LH pulse with the integrated limiter installed. Shots with arcing are indicated by a red circle. Shots without arcing are indicated by a blue asterisk. Vertical dashed lines indicate the start of a new run day. Overnight boronizations between run days are indicated by BZN.

\section{High Power Fast Ferrite Stub Tuner}

A prototype high power double-stub matching network has been developed to provide feedback-controlled impedance matching for the LH antenna on C-Mod. Stub tuners have been used extensively for impedance matching of ion cyclotron range of frequency (ICRF) antennas [15], but this technique has not been applied to LH antennas. A double stub tuner can be used to match any reflection coefficient outside the "forbidden region" (a circle on the Smith Chart centered on the real axis and tangent to the $\Gamma=1$ circle) [16]. The reflection coeffient can be reduced from $\Gamma$ to $\Gamma^{2}$ (from $\Gamma^{2}$ to $\Gamma^{4}$ in power) within the forbidden region.

The matching network consists of a compact double-stub tuner as shown in Figure 12. The waveguide used is conventional WR187 with an interior dimension of $4.755 \times 2.215 \mathrm{~cm}$. The two stubs are separated by $3 \lambda / 8$, which provides a good compromise between the size of the forbidden region and sensitivity of the matching network. Each stub is shorted at one end, and the electrical length can be varied by adjusting the magnetic field applied to a ferrite slab located inside the stub. The ferrite is a calcium vanadium doped garnet measuring $3 \times 1 \times 0.1 \mathrm{in}$. Neodymium permanent magnets provide a $0.4 \mathrm{~T} \mathrm{DC}$ bias field, and electromagnets are used to provide an offset in either direction. The electromagnets are 100 turn copper coils and can adjust the 


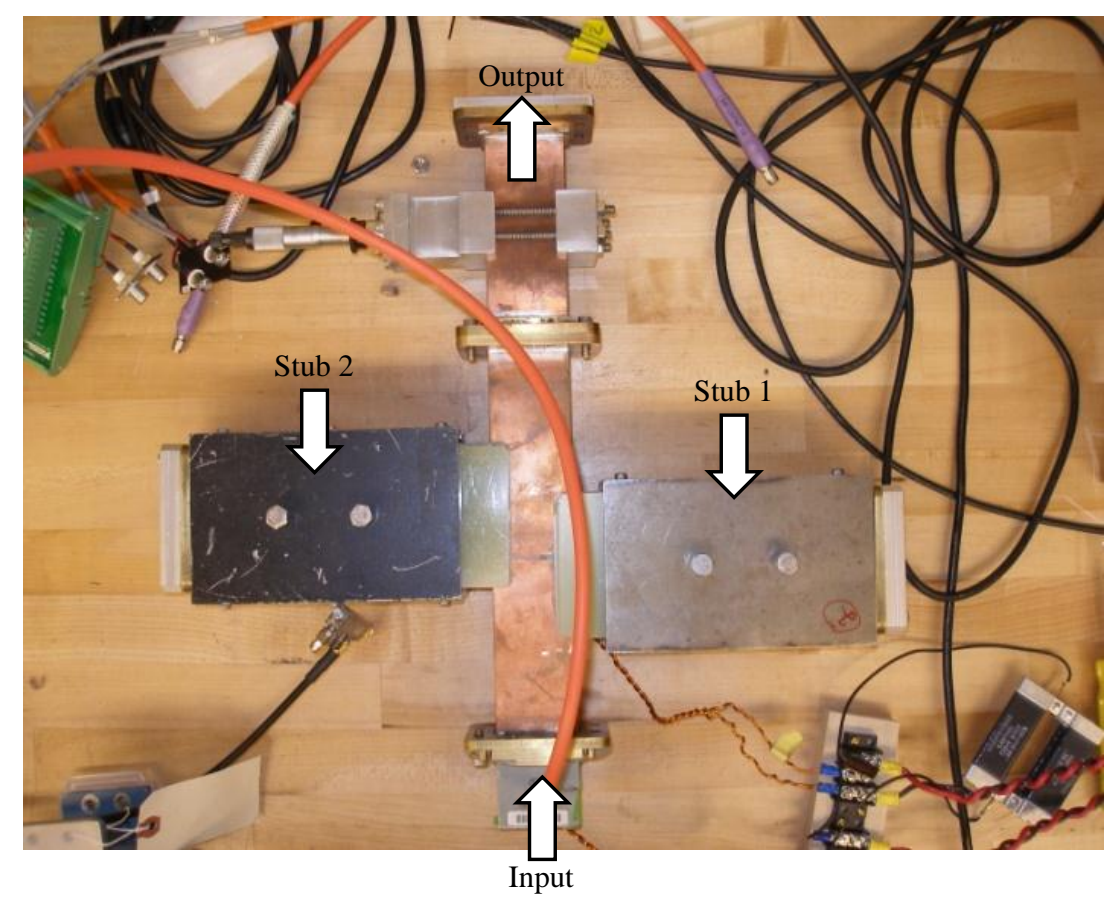

Figure 12. A photograph of the prototype high power double-stub fast ferrite tuner (FFT). The two stubs are separated by $3 \lambda / 8$. Need better picture!

magnetic field at the ferrite by $\pm 0.03 \mathrm{~T}$ by varying the coil current across a range of $\pm 3 \mathrm{~A}$. Low power testing shows that the stub length can be varied by greater than $180^{\circ}$ at $4.6 \mathrm{GHz}$.

High power tests show that the ferrite can withstand $170 \mathrm{~kW}$ of power without arcing. The top edges of each ferrite are rounded to ??? in to improve power handling by reducing electric field concentration at sharp edges. The ferrite in these tests was located on the center of the broad wall of the waveguide (in the region with the highest electric fields) and held in position by the permanent magnetic field. The magnetic field is sufficient to prevent movement of the ferrite during testing, although rough handling can cause the ferrite to become dislodged. A procedure has been developed to bond a metallic substrate to the bottom of the ferrite so it may be permanently soldered to the waveguide wall. Put specifics of ferrite bonding process here.

The length for each stub can be calculated analytically based on the measured complex reflection coeffient on the unmatched side of the FFT [17, 18]. Figure 13 shows the feedback loop for the FFT control system. A directional coupler on the unmatched side of the FFT samples the forward and reflected waves. The directional coupler on the matched side of the FFT can similarly be used to compute the proper stub lengths. A stub tuner can perturb the phase of the forward wave on the unmatched side of the tuning network. This presents a problem for use in a LHCD antenna because the launched $n_{\|}$spectrum depends on the phase shift between columns of the antenna. This effect can be corrected by measuring the phase of the forward wave with a directional 


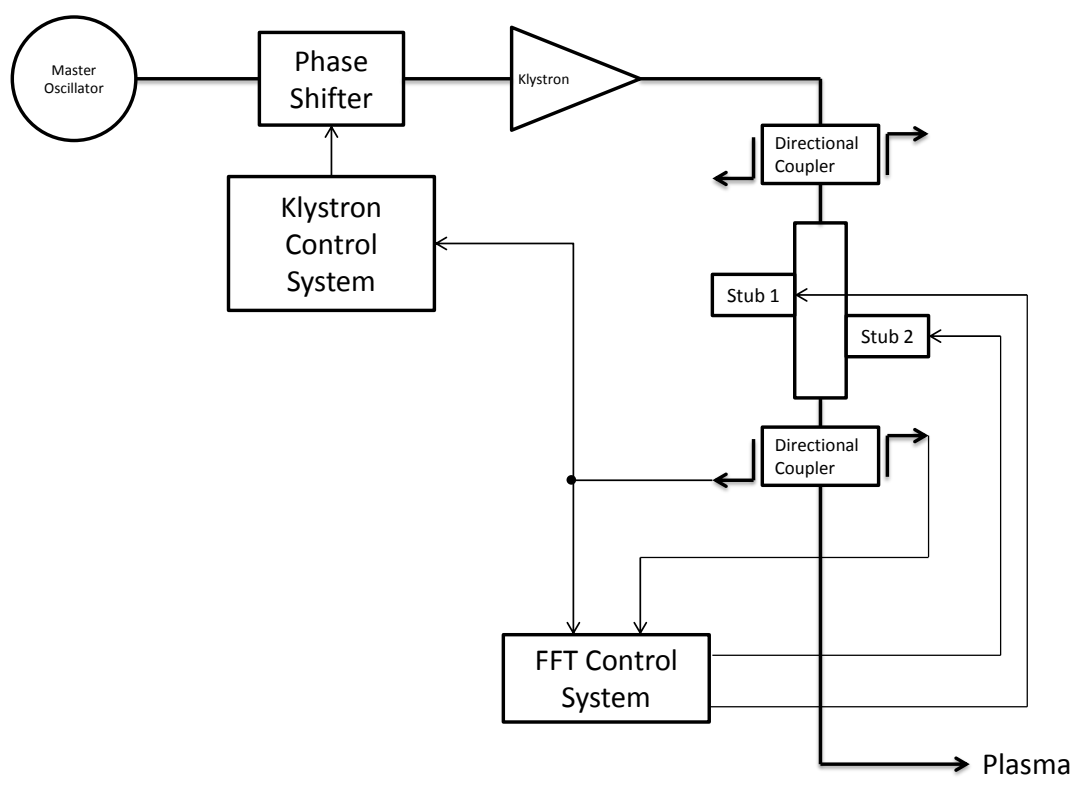

Figure 13. A schematic representation of the klystron and FFT control systems.

coupler on the unmatched side of the FFT and adjusting the phase of the klystron amplifier accordingly. Figure 13 shows the feedback loop of the klystron control system incorporates the phase perturbation introduced by the FFT.

\section{Future direction and conclusions}

This paper provides a summary of recent progress in LHCD technology at the MIT Plasma Science and Fusion Center. Research and development efforts have concentrated on improving the effectiveness of LHCD through design of an above mid-plane LH launcher, extension of the LHCD pulse length, and reduction of reflection coefficients. Future work will focus on installation of the additional source power, construction of the advanced LH3 antenna, and live testing of the double-stub FFT with a plasma loaded antenna.

Recent simulations [19] have shown that achieving ITER's steady-state goal may require substantial off-axis current drive produced by 20-40 MW of LHCD. Alcator CMods unique parameters provide a crucial test-bed for engineering and physics issues associated with the use of LHCD on ITER. The C-Mod research effort on LHCD is laying the groundwork needed for validated simulations which will allow reliable predictions of the performance of lower hybrid current drive systems in ITER, and its ability to access steady state regimes at $Q>5$. 


\section{Acknowledgments}

This work supported by USDOE awards DE-FC02-99ER54512 and DE-AC02$76 \mathrm{CH} 03073$.

\section{References}

[1] R. Aymar, P. Barabaschi, and Y. Shimomura. The ITER design. Plasma Physics and Controlled Fusion, 44:519-565, May 2002.

[2] Y. Shimomura, R. Aymar, V.A. Chuyanov, M. Huguet, H. Matsumoto, T. Mizoguchi, Y. Murakami, A.R. Polevoi, M. Shimada, ITER Joint Central Team, and ITER Home Teams. Iter-feat operation. Nuclear Fusion, 41(3):309, 2001.

[3] G.T. Hoang, A. Bécoulet, J. Jacquinot, J.F. Artaud, Y.S. Bae, B. Beaumont, J.H. Belo, G. BergerBy, Joāo P.S. Bizarro, P. Bonoli, M.H. Cho, J. Decker, L. Delpech, A. Ekedahl, J. Garcia, G. Giruzzi, M. Goniche, C. Gormezano, D. Guilhem, J. Hillairet, F. Imbeaux, F. Kazarian, C. Kessel, S.H. Kim, J.G. Kwak, J.H. Jeong, J.B. Lister, X. Litaudon, R. Magne, S. Milora, F. Mirizzi, W. Namkung, J.M. Noterdaeme, S.I. Park, R. Parker, Y. Peysson, D. Rasmussen, P.K. Sharma, M. Schneider, E. Synakowski, A. Tanga, A. Tuccillo, and Y.X. Wan. A lower hybrid current drive system for ITER. Nuclear Fusion, 49(7):075001, 2009.

[4] P. T. Bonoli, R. Parker, S. J. Wukitch, Y. Lin, M. Porkolab, J. C. Wright, E. Edlund, T. Graves, L. Lin, J. Liptac, A. Parisot, A. E. Schmidt, V. Tang, W. Beck, R. Childs, M. Grimes, D. Gwinn, D. Johnson, J. Irby, A. Kanojia, P. Koert, S. Marazita, E. Marmar, D. Terry, R. Vieira, G. Wallace, J. Zaks, S. Bernabei, C. Brunkhorse, R. Ellis, E. Fredd, N. Greenough, J. Hosea, C. C. Kung, G. D. Loesser, J. Rushinski, G. Schilling, C. K. Phillips, J. R. Wilson, R. W. Harvey, C. L. Fiore, R. Granetz, M. Greenwald, A. E. Hubbard, I. H. Hutchinson, B. LaBombard, B. Lipschultz, J. Rice, J. A. Snipes, J. Terry, S. M. Wolfe, and the Alcator C-Mod Team. Wave-Particle Studies in the Ion Cyclotron and Lower Hybrid Ranges of Frequencies in Alcator C-Mod. Fusion Science and Technology, 51(3):401-436, April 2007.

[5] S. Shiraiwa, O. Meneghini, R.R. Parker, G. Wallace, J. Wilson, I. Faust, C. Lau, R. Mumgaard, S. Scott, S. Wukitch, W. Beck, J. Doody, J. Irby, P. MacGibbon, D. Johnson, A. Kanojia, P. Koert, D. Terry, R. Vieira, and the Alcator C-Mod team. Design, and initial experiment results of a novel LH launcher on Alcator C-Mod. Nuclear Fusion, 51(10):103024, 2011.

[6] D. Moreau and T.K. N'guyen. Plasma physics. Proc. 6th Joint Kiev Conf., 1:225, 1984.

[7] J. Hillairet, D. Voyer, B. Frincu, O. Meneghini, A. Ekedahl, and M. Goniche. Modeling of lower hybrid antennas using the aloha code and comparisons with tore supra experiments. Fusion Engineering and Design, 84(2-6):953-955, 2009.

[8] S. Shiraiwa et al. submitted to Nuclear Fusion, 2012.

[9] A.D. Kanojia, G.M. Wallace, D.R. Terry, J.A. Stillerman, W.M. Burke, P.A. MacGibbon, and D.K. Johnson. Active control system upgrade design for lower hybrid current drive system on alcator c-mod. Fusion Engineering and Design, 87(12):1981 - 1984, 2012.

[10] A. Mizuhara. private communication, 2007.

[11] D.R. Terry et al. In "Proceedings of the Topical Meeting on the Technology of Fusion Energy", volume 56 of American Nuclear Society, pages 119-124, 2009.

[12] M. Grimes, D. Gwinn, R. Parker, D. Terry, and J. Alex. The Alcator C-Mod lower hybrid current drive experiment transmitter and power system. Proceedings of the $19^{\text {th }}$ Symposium on Fusion Engineering, pages 16-19, 2002.

[13] R.T. Mumgaard, S.D. Scott, S. Shiraiwa, G.M. Wallace, and R.R. Parker. MSE constrained magnetic reconstructions of Lower Hybrid Current Drive on Alcator C-Mod. Bull. Am. Phys. Soc., 57(12):181, 2012.

[14] M. Preynas, A. Ekedahl, N. Fedorczak, M. Goniche, D. Guilhem, J.P. Gunn, J. Hillairet, 
X. Litaudon, J. Achard, G. Berger-By, J. Belo, E. Corbel, L. Delpech, T. Ohsako, and M. Prou. Coupling characteristics of the iter-relevant lower hybrid antenna in tore supra: experiments and modelling. Nuclear Fusion, 51(2):023001, 2011.

[15] R.I. Pinsker. Development of impedance matching technologies for icrf antenna arrays. Plasma Physics and Controlled Fusion, 40(8A):A215, 1998.

[16] D.M. Pozar. Microwave Engineering. John Wiley \& Sons, Inc., 1998.

[17] Y. Lin, J.A. Stillerman, A. Binus, A. Parisot, and S. Wukitch. Digital real-time control for an ICRF fast ferrite tuning system on Alcator C-Mod. Fusion Engineering and Design, 83(23):241 $-244,2008$.

[18] Y. Lin, A. Binus, and S.J. Wukitch. Real-time fast ferrite ICRF tuning system on the Alcator C-Mod tokamak. Fusion Engineering and Design, 84(1):33 - 37, 2009.

[19] F. Poli et al. submitted to Physics of Plasmas, 2012. 